\title{
Towards an Affective Computing Feedback System to Benefit Underserved Individuals: An Example Teaching Social Media Skills
}

\author{
Mohammad Nasser Saadatzi ${ }^{1}$, Karla Conn Welch ${ }^{1}$, \\ Robert Pennington ${ }^{2}$, and James Graham ${ }^{1}$ \\ ${ }^{1}$ University of Louisville, Department of Electrical and Computer Engineering, \\ 448 Lutz Hall, Louisville, KY 40292, USA \\ ${ }^{2}$ University of Louisville, Department of Special Education, \\ College of Education and Human Development, Louisville, KY 40292, USA \\ \{mn.saadatzi, karla.welch, robert.pennington, \\ james.graham\} @louisville.edu
}

\begin{abstract}
Researchers have suggested that the use of technology may be effective during the instruction of a variety of academic and communication skills for individuals with disabilities [1,2]. Also, the design of affect-sensitive interactions between humans and technology, a research area known as affective computing, is an increasingly important discipline in the humancomputer interaction (HCI) and human-robot interaction (HRI) communities. Physiological signals could be used to determine which affective states are involved in HCI and HRI for a broad section of the population but may have increased utility for individuals with social or intellectual impairments. Therefore, employing affect-sensitive technologies in intervention sessions may provide a means to make strides in appropriate social interaction skills and other deficits, but further research is necessary to understand why these methods are successful and what applications are most useful for different individuals.
\end{abstract}

Keywords: affective computing, autism, intellectual disabilities, social media.

\section{Introduction}

Since the recognition of emotion and affective expressions plays a critical role in decision-making, learning, and other cognitive functions; human interaction with technology will likely improve if those interactions also involve systems that facilitate affect recognition. Affect-sensitive systems may be useful for individuals with autism spectrum disorders (ASD) and other developmental disorders, who often exhibit deficits in emotion comprehension. For individuals with intellectual disabilities (ID), an often underserved population, affective computing could allow for similar instruction benefits on social communication training and increasing the presentation rate of skills. These systems could potentially serve to ameliorate deficits in emotional 
understanding by augmenting this impaired skill area during a variety of instructional interactions. In this paper, we will first provide an overview of the benefits of monitoring physiological signals during interactive activities followed by a description of our preliminary work in this area. Second, we will describe a pilot investigation of the use of a robot to teach social media skills. The resulting data from these studies serve to provide a foundation for the future development of successful affect-sensitive closed-loop interaction.

Physiological signals can be used to monitor changes in affective responses. These signals offer compelling advantages in evaluating affective responses as they are continuously available and permit the gathering of rich data in the face of potential communicative limitations. Physiology-based affective computing also can aid in teaching social interaction skills by monitoring the impact of particular social stimuli on an individuals' physiological signals and subsequently adjusting instruction to delivering prompts to facilitate successful interactions.

\section{Background}

A computer that can detect the affective states of a student and interact with him/her based on such perception could have a wide range of potential impacts. Complex social stimuli, sophisticated teacher-learner interactions, and unpredictable situations could be gradually, but automatically, introduced when the computer has the knowledge that the student is comfortable or not anxious at a certain level of interaction dynamics for a reasonably long period of time. However, the current computer-assisted therapeutic tools for children with ASD and/or ID do not possess the ability of deciphering the affective cues of the children, which could be critical given that affective factors have significant impacts on the intervention practice.

Computers and robots have been used to teach basic social interaction skills to young children with ASD when using turn-taking and imitation games [3]. The origin of robotic endeavors in ASD therapy can be traced to the 1970s through a seminal work done in Edinburg [4], and computer [5, 6] and robotic [7] approaches to autism diagnosis and treatment have continued through present-day research. HRI may work well for an initial intervention to remove the difficulties related to direct human interaction that is part of typical therapy settings. Robots should not be considered an isolating agent, because dyadic communication accomplished between an individual and a robot can lead into triadic communication including a therapist, caregiver, or peer and in due course potentially accomplish the intervention goals of developing social communication skills between the individual and another person [8]. By employing affect-sensitive technologies, HCI and HRI therapeutic tools can partially automate time-consuming behavioral therapy sessions, allow for an increased number of exposures to a teaching item, and may allow intensive intervention to be conducted at home.

However, despite advances, to our knowledge current computer and robotic treatments available for children with developmental disorders do not provide autonomous capabilities and suffer from a lack of enough adaptation to each user's 
(dis)abilities. Since ASD and ID occur across a continuum of severity, interventionists are faced with a user group with substantial heterogeneity [9]. Therefore, it is unlikely that one system can be used generically to satisfy the needs of all users. Therefore, we believe that customizable, adaptable feedback systems capable of eliciting specific basic interaction skills are needed. Thus, our final goal is to develop an affectsensitive feedback system which adapts with respect to each individual's needs and continuously modifies the interaction based on the user's performance as well as progress.

The experimental setup for our system is shown in Figure 1. The design allows for a student to participate in an interactive session (e.g., session focused on social skills, storytelling, etc.) with a NAO robot (aldebaran-robotics.com) while his/her physiological data are measured using wearable biofeedback sensors from Biopac (biopac.com). Biopac filters and digitizes the physiological signals, and then transfers them through an Ethernet link to the main computer. A therapist with experience in working with individuals with intellectual disabilities and the participant's teacher also observe the sessions from the view of the video camera and observing how the task progressed on separate monitors.

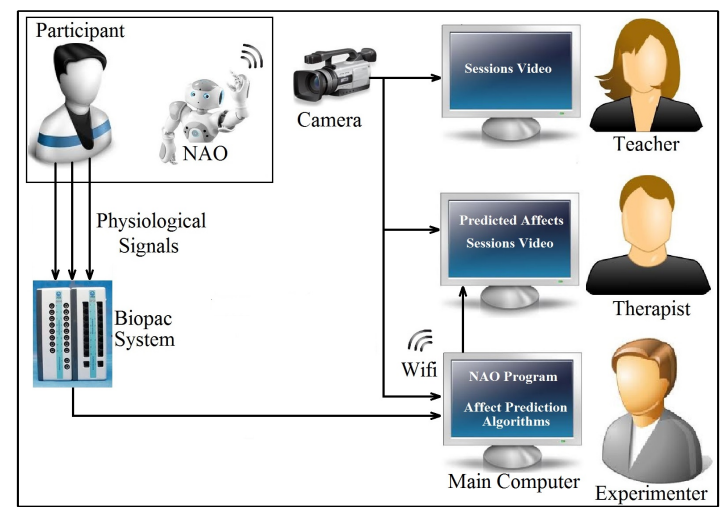

Fig. 1. Experimental setup for the affect-sensitive closed-loop interaction

\subsection{Physiology-Based Affective Modeling for Children with ASD}

In previous pilot work, six adolescents diagnosed with ASD played computer games that varied in difficulty to elicit emotional responses while simultaneous clinical observation, performance characteristic/evaluation, and physiological data were collected. A comprehensive set of relevant physiological features were derived from the physiological signals, and physiology-based affective models were developed from readily observable behaviors with presumable underlying affective states. A Support-vector Machines (SVM) approach was applied to create an affective model for recognizing behavioral responses from the HCI data, which yielded reliable prediction with approximately $83 \%$ success [10]. Such prediction accuracy is considered high even among typical adults [11, 12]. A second phase of this research employed the affective models during HRI wherein the subjects tossed Nerf balls at a 
moving robotic arm with a basketball hoop attached to its end-effector. The robot learned preferences based on the predicted enjoyment level of children with ASD and selected an appropriate movement in real-time. Each participant completed two sessions, (1) non-affect sensitive and (2) affect sensitive. Results indicated a highly significant difference between conditions, with the affect-sensitive modeling improving performance as well as enhancing enjoyment of the task [13]. This work suggested observations during HCI could be successfully applied to HRI; thus expanding the possible tasks available to develop affective models. This work is the first time that the impact of affect-sensitive closed-loop interaction between a robot and children with ASD had been demonstrated experimentally. While this previous work was restricted to goal-oriented tasks, it demonstrated the feasibility of the design for monitoring affective responses in children with ASD in social-communication tasks. In the next section we briefly present our latest preliminary work to demonstrate the feasibility as well as the likelihood of success of our proposed affectsensitive system.

\section{Two Preliminary Investigations}

\subsection{Affective Reactions to Social Interactions Set in Virtual Reality for Teenagers with ASD and Typically-Developing Matched Peers}

This study (set up and participant characteristics described in [14, 15]) examined affective and physiological variation in response to manipulated social parameters (i.e., eye gaze and social distance) during social interaction in Virtual Reality (VR) for both ASD and Typically-developing (TD) children. Thirteen matched-pairs of children with ASD and TD (age 13-17 years) listened and watched virtual human characters (i.e., avatars) who displayed different eye gaze patterns and stood at different distances while telling a personal story. Physiological responses and ratings from clinical observations resulted in significant differences to changes in social experimental stimuli. The reported change in affect are similar to observations in social anxiety research of typical adults in real-world settings [16, 17, 18] but have now been examined with observations and physiological signals for ASD and TD children in HCI. Establishing realistic interactions builds a basis for creating more complex settings for HCI intervention and will guide design of real-world social robots for embodied social communication intervention.

Both the TD and ASD group's physiological signals showed significant changes to trials rated as eliciting "low anxiety" (LA) versus "high anxiety" (HA) according to the clinical observer label (COL). For example, the phasic response rate of skin conductance, measured in peaks per minute (ppm), reflected both the ASD and TD group had a significant increase between trials rated as LA and HA, for trials in which the social distance parameter was set to Invasive for all variations of the eye gaze parameter (as shown in Table 1). As anxiety increased in these conditions, the phasic response rate significantly increased within the groups; and between the groups the ASD group showed more peaks per minute during LA and HA. Therefore, even for lower anxiety situations, children with ASD showed a significantly higher affective 
response. Furthermore, this work highlighted that, although group affective models from a small number trials from each participant have been used to evaluate affective states of typical adults [19], separate ASD and TD models might be necessary considering the significant physiological differences detected between the groups.

Table 1. Listed are results of phasic response rate from the skin conductance signal compared between trials labeled as LA and HA. The trials considered were ones in which the social distance parameter was set to Invasive for all variations of the eye gaze parameter. Significant difference, ${ }^{*} p<0.05$.

\begin{tabular}{ccclc}
\hline COL & $\begin{array}{c}\text { ASD group } \\
\text { ppm mean }\end{array}$ & $\begin{array}{c}\text { TD group } \\
\text { ppm mean }\end{array}$ & Group Differences \\
\hline LA & 4.43 & 3.23 & $p$-value & $0.0227^{*}$ \\
HA & 5.80 & 4.46 & $p$-value & $0.0286^{*}$ \\
$t$-value & -2.18 & -2.33 & & \\
$p$-value & $0.0311^{*}$ & $0.0211^{*}$ & & \\
\hline
\end{tabular}

Two machine-learning algorithms - SVM and K-nearest neighbor (KNN) - were implemented to compare prediction accuracies of the affective models for identifying anxiety. The KNN algorithm for best accuracy used 6 neighbors, square euclidean distancing, and single nearest neighbor as a tiebreaker. The SVM algorithm used a linear kernel function and a least squares method. The accuracy results (shown in Table 2) indicate low reliability for either algorithm to predict high vs. low anxiety based on the data merged by group. An accuracy above $70 \%$ is considered high based on previous research on typical adults $[12,19,20,21]$; however, even the TD group did not yield over $70 \%$ on this data. Although group models have been used for detecting stress situations for typical adults (see Table 3 for comparison work), combining ASD subjects as a group may not yield accurate results.

Table 2. Prediction accuracies for anxiety based on a ASD and TD group models, comparing SVM and KNN

\begin{tabular}{ccccc}
\hline Algorithm & $\begin{array}{c}\text { ASD group } \\
\text { Anxiety Accuracy (\%) }\end{array}$ & $\begin{array}{c}\text { TD group } \\
\text { Anxiety Accuracy (\%) }\end{array}$ & \multicolumn{2}{c}{ Group Differences } \\
\hline SVM & 56.6978 & 65.1090 & $p$-value & $0.0294^{*}$ \\
KNN & 54.3478 & 68.3230 & $p$-value & $0.000259^{*}$ \\
$p$-value & $0.5796, \mathrm{~ns}$ & $0.3588, \mathrm{~ns}$ & & \\
\hline
\end{tabular}

From the analysis shown in Table 2, changing up the algorithm will likely not gain accuracy. Further analysis is needed to determine the accuracies of building a model from a smaller set of physiological signals. We need to go for a smaller set of features and need to consider an individual-specific approach. Although group analysis would allow the models to grow at a faster rate by compounding data collection, unique 
Table 3. Ref. [19] used group models of TD subjects based on 11 physiological features. Ref. [13] and the current VR study used 53 physiological features. This comparison might suggest that individual analysis, which has seemed useful in TD work [12], may be particularly needed for ASD groups. Also, analysis of smaller sets of features might increase the accuracy of predicting affect based on the VR data.

\begin{tabular}{|c|c|c|c|c|c|c|c|}
\hline $\begin{array}{l}\text { Compa } \\
\text { rison } \\
\text { Work } \\
\end{array}$ & $\begin{array}{l}\text { Affect to } \\
\text { Predict }\end{array}$ & $\begin{array}{l}\text { Model } \\
\text { Type }\end{array}$ & Subjects & Samples & Features & Algorithm & Accuracy \\
\hline [\#\#] & Stress & Group & $\begin{array}{l}32 \mathrm{TD} \\
\text { Adults }\end{array}$ & 192 & 11 & SVM & $90.1 \%$ \\
\hline [\#\#] & $\begin{array}{l}\text { anxiety } \\
\text { engagement } \\
\text { Liking }\end{array}$ & Individual & $\begin{array}{c}6 \\
\text { teenager } \\
\text { s with } \\
\text { ASD } \\
\end{array}$ & $\begin{array}{c}85 \\
\text { each }\end{array}$ & 53 & SVM & $\begin{array}{c}\text { Overall } \\
\text { average } \\
82.9 \%\end{array}$ \\
\hline $\begin{array}{c}\text { Curren } \\
\text { t VR } \\
\text { Study }\end{array}$ & Anxiety & Group & $\begin{array}{c}13 \text { pairs } \\
\text { of TD } \\
\text { and ASD } \\
\text { teenager } \\
\mathrm{s}\end{array}$ & $\begin{array}{c}312 \\
\text { in each } \\
\text { group }\end{array}$ & 53 & KNN & $\begin{array}{l}\text { ASD: } 56.7 \% \\
\text { TD: } 65.1 \% \\
\text { ASD: } 54.3 \% \\
\text { TD: } 68.3 \%\end{array}$ \\
\hline
\end{tabular}

analysis may prove more beneficial for longitudinal applications. With making individual models, the challenge is collecting enough samples. When thinking about making an affect-sensitive feedback system to use in an educational setting or intervention setting for a long time, the premise is there to collect many samples. However, on day one, if you cannot apply an accurate group model, you are starting from scratch with each student. Those are some of the challenges we plan to address in future work

Even so, this work is the first time a large set of physiological features have been examined for a sizeable group of ASD and TD children during social situations presented on a VR platform for elicitation of affective states. So far the results show the VR system provokes variations in both affective ratings and physiological signals to changes in experiment conditions for both children with autism and typical children. This work provides a step towards the development of future interventions using affect-sensitive technologies for the ASD population, but additional analysis remains to be examined. From these initial results, we will examine possible improvements by focusing on a smaller set of features and/or collecting more samples for an individual-specific approach.

\subsection{Case Study on Social Media}

In this pilot investigation, we evaluated the effects of robot delivered instruction on text messaging responses of a 19-year old male with Down Syndrome. The young man attended a public transition classroom for individuals with intellectual disabilities 
that was housed on a university campus. The student's teacher sought assistance in expanding his texting repertoire as his typical text messages consisted of one or two unidirectional statements about a preferred musical artist (e.g., I like Selena Gomez. She is cute.). First, we approached a class of undergraduate students in a teacher education program, similar in age to the students in the transition program, to identify age appropriate texting responses. Students in the class were asked to consider the appropriateness of responses that contained three key components; (a) a greeting (e.g., hey), (b) a statement about an event in the students life (e.g., went to the movies last night), and (c) a closing (e.g., later). After considering the students' feedback in the selection of instructional targets, we designed an instructional protocol that would be used to program a humanoid robot to teach the student the new responses.

The robot used in this study to deliver the instructions is a NAO robot. NAO is a knee-size humanoid robot that has a variety of sensors and capabilities including digital cameras, speakers, microphone arrays, tactile sensors, text-to-speech, and voice recognition as well as face and object detection. At the onset of each teaching session, the robot delivered an attention cue (i.e., Look at me), and waited for the student to respond. If NAO did not detect the student's attention response (i.e., face oriented towards NAO), it repeated the cue. Once the student emitted the attention response, NAO vocally prompted the student to ready his phone for texting and indicate preparedness by stating, "Ready". Following the recognition of the student's response, NAO started instruction on the texting components.

During instruction, the robot used simultaneous prompting procedures [22]. Simultaneous prompting is an errorless teaching procedure that involves the delivery of a controlling prompt immediately following a discriminative stimulus (e.g., teacher directive) during teaching trials. Additionally, the transfer of stimulus control is assessed through the administration of daily probe trials prior to training. First, NAO instructed the student to write a greeting, and then immediately provided a prompt (e.g., "Write, Hey"). Once the student vocally indicated that he was finished writing, NAO nodded its head in approval and directed the student to write about a personal event. Again it delivered a prompt (e.g., I played basketball yesterday) and waited for the student to respond. The researchers had previously identified accurate prompts through interviews with the teacher and her student. Again, NAO waited for the student to indicate completion and subsequently directed and prompted the student to write a closing (e.g., Later, Goodbye). Upon completion of all three steps, the robot delivered vocal praise and offered to perform a song that previously had been indicated by the student to be a preference.

During baseline sessions and immediately prior to treatment sessions, the researchers conducted data probes. During the probe, the student's teacher instructed him to text the researcher he was familiar with through interaction in a previous study. The teacher informed the student that she could help with spelling only. Upon completion of the text, the teacher prompted the student to hit send and wait for the researcher's response. The researcher responded immediately with a general statement (e.g., cool, awesome). Data were collected on the number of components used in the student's text responses (i.e., greeting, personal statement, closing). 
Data suggest that the student met criterion (i.e., including all three components across 3 consecutive probes) after seven teaching sessions. During baseline sessions, texted responses generally included statements about a musical group (i.e., I like Selena Gomez, Katie Croom love her deepest heart and soul). Following intervention, the student texted responses that contained all three targeted elements (i.e., Hi We won our game last night Good bye; Hi, we have a basketball game on Wednesday Good bye). Since teaching sessions generally lasted for approximately four minutes, the student acquired the skills in less than thirty minutes of instruction. Though these preliminary data hint at the potential of robot delivered instruction as an effective an efficient means of teaching news skills to students with disabilities, it is important to note that several changes during instruction severely limit the generalizability of our findings. First, on several occasions the research team had to prompt the student to speak clearly or repeat their responses. Second, NAO did not provide explicit feedback on the students' texts during instruction. The team refined instructional procedures to include a teacher feedback component, whereas the teacher guided the student to graph the number of elements used following each probe session.

One of the goals of our research team is to design NAO's vocalization and movements natural enough so that the user conceives the robot as an animate entity rather than a toy. At the same time, the interactions should be as simple as possible in order to avoid delivering overwhelming amount of details and information, to ensure minimal unexpected interactions, increased anxiety, and session interruption. The architecture of our program for NAO, to perform its tasks autonomously and desirably, has several layers that work in parallel. One of these layers is responsible for deciding and enforcing NAO's movements during the interaction. This layer determines proactive and reactive movements carried out at one time based on the type of the instructions being delivered at the time, and the participant's performance. This layer assigns each part of NAO a relevant motion. For example, NAO's head normally track's the participant face during the whole interaction unless NAO is to express confirmation, surprise, etc. by its head. The same process is true for the rest of NAO's body. Several body motions were designed for NAO, using NAO's 25 degrees of freedom, to express different gestures. This layer picks among this library of motions based on the interaction statistics.

The student's story telling skills have thus far shown mild improvement, but the results are preliminary. This case study has served to provide vital information on best practices for a longitudinal study on the use of an affective-sensitive feedback system utilizing a robot to teach social media skills. The specific instruction focuses on teaching appropriate parts of a story, constructed within text messages. We also will evaluate as to whether the newly acquired text message skill translates to improvements in Facebook status updates. These skills may enhance the quality of life and potentially increase social networks for individuals with ASD/ID. Furthermore, we believe incorporating affect-sensitivity into such instruction may establish instructional technology that more closely reflects human teacher-student relationships. Ultimately we seek to provide underserved individuals with a means to learn how to interact more fully in the social world. 


\section{Discussion}

The impact of research in the area of robot affective computing has broad potential. For example, in the current investigation, we programmed a robot to perform instructional tasks that involved assessing the occurrence a student's attention response while delivering instruction on age-appropriate and socially-relevant tasks. During final instructional sessions, the student interacted with the robot without additional teacher supports. This allowed the classroom teacher to work with other students while the targeted student engaged in meaningful instruction with the robot. These findings suggest that robots could potentially be programmed to teach a variety of discrete and chained tasks, thus reducing student to teacher ratios and increasing learning opportunities during teaching sessions.

These findings also direct us to consider alternate applications for robot-based affective computing. Robot instructors could easily be designed to teach employment related tasks (e.g., interviewing, talking to coworkers) in less aversive teaching contexts. Learners with ASD may benefit from the lack of prosody within digital speech and the absence of a requirement to read facial expressions during social communication tasks. Our research team is working on developing sophisticated tools to increase the responsivity of robot instructors to the needs of persons with ASD. Currently, we have proposed the use of autonomic sensors to guide the selection of instructional targets by robot instructors. In other words, the robot will change the difficulty of instructional tasks in response to changes in students' physiological markers of stress. This research is significant in light of data [23] suggesting that students often engage in problem behavior to escape a variety of instructional tasks.

\section{References}

1. Chen, S.H., Bernard-Opitz, V.: Comparison of personal and computer-assisted instruction for children with autism. Mental Retardation 31, 368-376 (1993)

2. Pennington, R.: Computer-assisted instruction for teaching academic skills to students with autism spectrum disorders: A review of literature. Focus on Autism and Other Developmental Disabilities 25, 239-248 (2010)

3. Dautenhahn, K., Werry, I.: Towards interactive robots in autism therapy: Background, motivation and challenges. Pragmatics Cognition 12, 1-35 (2004)

4. Saadatzi, M.N., Welch, K.C., Graham, J.: Robotic assistance for individuals with Autism: A survey of current technology and insight into future directions. In: ISCA International Conference on Computer Applications in Industry and Engineering (2012)

5. Bernard-Opitz, V., Sriram, N., Nakhoda-Sapuan, S.: Enhancing social problem solving in children with autism and normal children through computer-assisted instruction. Journal of Autism and Developmental Disorders 31(4), 377-384 (2001)

6. Parsons, S., Mitchell, P., Leonard, A.: Do adolescents with autistic spectrum disorders adhere to social conventions in virtual environments? Autism 9(1), 95-117 (2005)

7. Scassellati, B., Admoni, H., Matarić, M.: Robots for Use in Autism Research. Annual Review of Biomedical Engineering 14 (2012) 
8. Michaud, F., Theberge-Turmel, C.: Mobile robotic toys and autism. In: Dautenhahn, K., Bond, A.H., Canamero, L., Edmonds, B. (eds.) Socially Intelligent Agents: Creating Relationships with Computers and Robots, pp. 125-132. Kluwer, Norwell (2002)

9. Weir, S., Emanuel, R.: Using Logo to Catalyse Communication in an Autistic Child: Department of Artificial Intelligence (1976)

10. Conn, K., Liu, C., Sarkar, N., Stone, W., Warren, Z.: Towards Affect-sensitive Assistive Intervention Technologies for Children with Autism. In: Jimmy, O. (ed.) Affective Computing: Focus on Emotion Expression, Synthesis and Recognition, ARS/I-Tech Education and Publishing, Vienna (2008)

11. Picard, R.W., Vyzas, E., Healey, J.: Toward machine emotional intelligence: analysis of affective physiological state. IEEE Transactions on Pattern Analysis and Machine Intelligence 23(10), 1175-1191 (2001)

12. Rani, P., Liu, C., Sarkar, N., Vanman, E.: An empirical study of machine learning techniques for affect recognition in human-robot interaction. Pattern Analysis and Applications 9(1), 58-69 (2006)

13. Liu, C., Conn, K., Sarkar, N., Stone, W.: Online Affect Detection and Robot Behavior Adaptation for Intervention of Children with Autism. IEEE Transactions on Robotics 24(4), 883-896 (2008)

14. Welch, K.C., Lahiri, U., Warren, Z., Sarkar, N.: An Approach to the Design of Socially Acceptable Robots for Children with Autism Spectrum Disorders. International Journal of Social Robotics 2(4), 391-403 (2010)

15. Welch, K.C., Lahiri, U., Liu, C., Weller, R., Sarkar, N., Warren, Z.: An Affect-sensitive Social Interaction Paradigm utilizing Virtual Reality Environments for Autism Intervention. In: Jacko, J.A. (ed.) HCI International 2009, Part III. LNCS, vol. 5612, pp. 703-712. Springer, Heidelberg (2009)

16. Argyle, M., Dean, J.: Eye-contact, distance and affiliation. Sociometry 28(3), 289-304 (1965)

17. Schneiderman, M.H., Ewens, W.L.: The cognitive effects of spatial invasion. Pac. Sociology Rev. 14(4), 469-486 (1971)

18. Sommer, R.: The distance for comfortable conversations: a further study. Sociometry 25(1), 111-116 (1962)

19. Zhai, J., Barreto, A.: Stress Detection in Computer Users through Noninvasive Monitoring of Physiological Signals. Biomedical Science Instrumentation 42, 495-500 (2006)

20. Picard, R.W., Vyzas, E., Healey, J.: Toward machine emotional intelligence: analysis of affective physiological state. IEEE Transactions on Pattern Analysis and Machine Intelligence 23(10), 1175-1191 (2001)

21. Nasoz, F., Alvarez, K., Lisetti, C., Finkelstein, N.: Emotion recognition from physiological signals for presence technologies. International Journal of Cognition, Technology, and Work-Special Issue on Presence 6(1), 4-14 (2003)

22. Gibson, A.N., Schuster, J.W.: The use of simultaneous prompting for teaching expressive word recognition to preschool children. Topics in Early Childhood Special Education 12, 247-267 (1992)

23. Iwata, B.A., Pace, G.M., Dorsey, M.F., Zarcone, J.R., Vollmer, T.R., Smith, R.G., Rodgers, T.A., Lerman, D.C., Shore, B.A., Mazeleski, J.L., Goh, H., Cowdery, G.E., Kalsher, M.J., McCosh, K.C., Willis, K.D.: The functions of self-injurious behavior: An experimental epidemiological analysis. Journal of Applied Behavior Analysis 27, 215-240 (1994) 